\title{
Infrared thermography applied to the study of heated and solar pavement: from numerical modeling to small scale laboratory experiments
}

\author{
N. Le Touz ${ }^{\mathrm{a}, \mathrm{b}}, \mathrm{T}$. Toullier ${ }^{\mathrm{a}, \mathrm{b}}$, and J. Dumoulin ${ }^{*}, \mathrm{a}, \mathrm{b}$ \\ aIFSTTAR, COSYS-SII, Route de Bouaye - CS4, F-44344, Bouguenais, France \\ ${ }^{b}$ Inria, I4S Team, Campus de Beaulieu, F-35042 Rennes, France
}

\begin{abstract}
The present study addresses the thermal behaviour of a modified pavement structure to prevent icing at its surface in adverse winter time conditions or overheating in hot summer conditions. First a multi-physic model based on finite elements method was built to predict the evolution of the surface temperature. In a second time, laboratory experiments on small specimen were carried out and the surface temperature was monitored by infrared thermography. Results obtained are analyzed and performances of the numerical model for real scale outdoor application are discussed. Finally conclusion and perspectives are proposed.
\end{abstract}

Keywords: Hybrid solar road, Finite Element Modeling, Heat transfer, Infrared thermography calibration

\section{NOMENCLATURE}
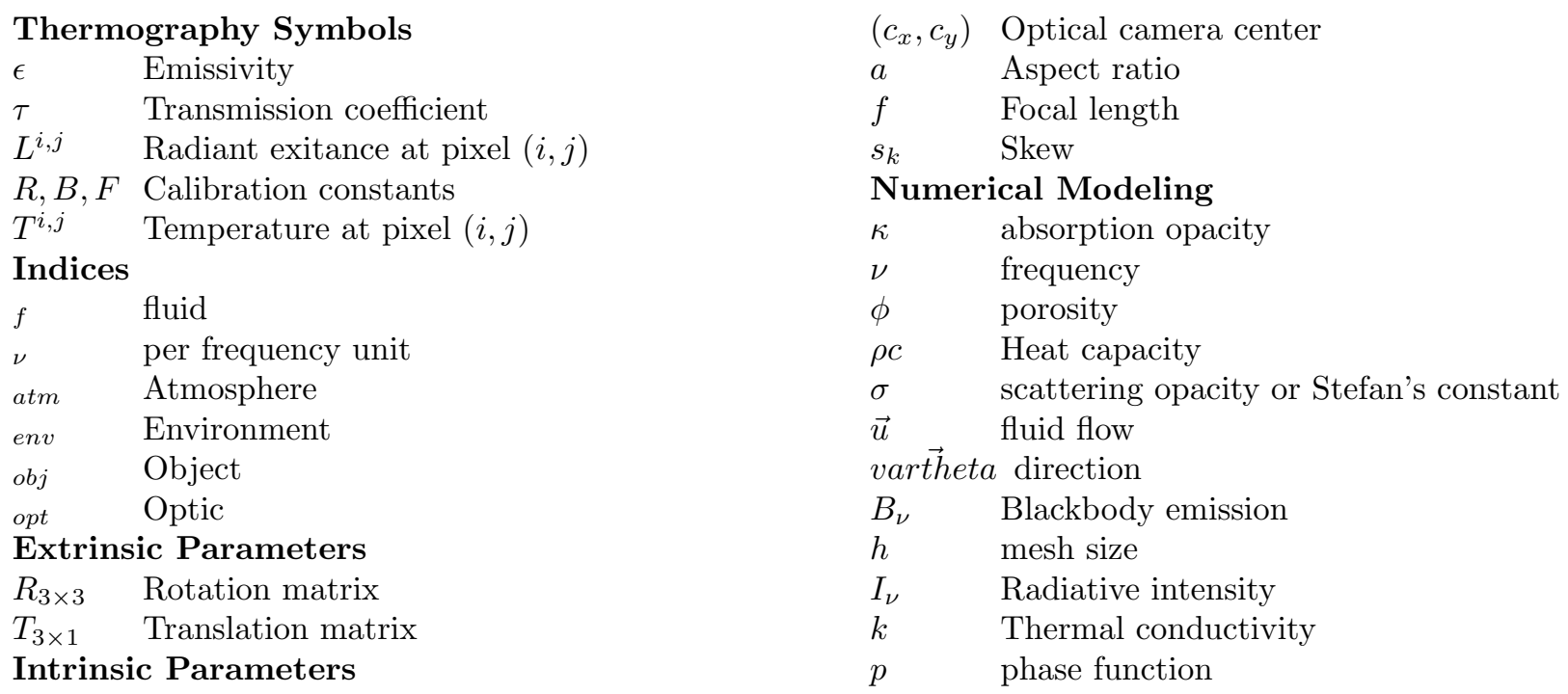

\section{INTRODUCTION}

In the context of the energy transition, that is to say energy saving and decreasing of fossil energy consumption, numerous studies and developments have led to the use of renewable energy (water, wind, solar, waves). Moreover, with the urbanization growth and the increasing need of road infrastructures efficient regardless of the weather conditions, road structures are the topic of research works.

Roads are subject to two phenomenon which can lead to energy recovery: the rolling of vehicles at the surface of the pavement and the contribution of solar radiation. ${ }^{1}$ Some solutions have been studied to collect

*jean.dumoulin@ifsttar.fr; phone 332 4084-5624; fax 332 4084-5998; www.ifsttar.fr 
in part these energy sources. Thus, the energy coming from the strain carried on by trucks and kinetic energy can be converted to electric energy with piezoelectric generators, ${ }^{2,3}$ or electromagnetic generators. ${ }^{2,4}$

Another way to collect energy from roads is to use solar radiation with collectors. These collectors can be photovoltaic systems converting thermal energy into electric energy ${ }^{1}$ or pipes located below the pavement in which a heat transfer fluid flows. ${ }^{5,6}$ Alternatively, it is possible to add a semi-transparent pavement. Moreover, these methods for energy recovery have the advantage to decrease the surface temperature during summer. Indeed, this temperature can reach $70^{\circ} \mathrm{C} .{ }^{7}$ Decreasing this surface temperature allows to reduce rutting provoked by the loss of bituminous materials rigidity. During winter, the opposite effect can be obtained: the flow of a warm fluid allows to heat the surface of the pavement, and so to avoid ice formation. ${ }^{8,9}$

To optimize the energy recovery capacity, without high changes in building methods, we study in this paper a recovery method based on a heat transfer fluid flowing in a porous layer, located below the pavement layer on which vehicles pass along. Then we evaluate the energy gain provided by the use of a semi-transparent layer as a pavement layer.

In this context, a numerical modelization was studied and developed to solve this coupled problem including thermal diffusion, hydraulic convection and radiative transfers. After an introduction of the concept of this solar-hybrid road, we present the studied modelization based and developed with the finite element method. Then, solving methods are coupled to realize an evaluation of the energetic performances on a study case. An infrared camera is implemented on a mock-up made in our laboratory and used as a test bench in this study. The purpose of such instrumentation is to show the possibilities of a non destructive, low cost and robust thermal monitoring solution. The models used to convert the numerical values of the camera to temperature and the methods used to compare the modelization with the measurements are introduced. Finally, the effect of adding a semi-transparent layer for the pavement is discussed, then conclusions and perspectives are presented.

\section{CONCEPT OF MODIFIED ROAD STRUCTURE AND NUMERICAL MODELING}

\subsection{Concept of modified structure}

We introduce in this section the concept of multilayer hybrid solar road for which the two first layer from the surface are subject to changes. Such a road is depicted in figure 1.

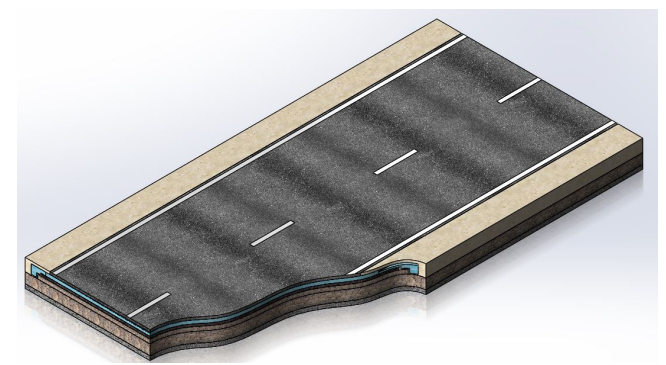

Figure 1: Schematic view of a modified structure from a $3 \mathrm{D}$ CAD

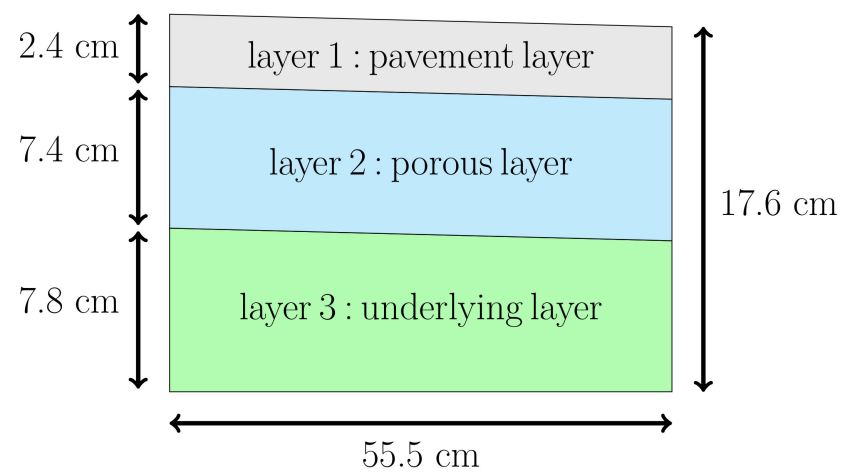

Figure 2: Sectional schematic view of modified road structure

Figure 2 represents a sectional view of the studied road. Layer 1 is semi-transparent or opaque. Layer 2 is porous: a fluid flows inside along the width of the road, with the effect of the imposed tilt. Layers 3 is opaque.

The porous layer presents a slope which allows the fluid to flow. A part of the solar incident direct heat flux during summer can be collected. On the contrary, for cold conditions, fluid flow allow to heat the surface of the pavement and also to prevent from icing. ${ }^{8}$ 
This structure is submitted to 3 thermophysical phenomenon: heat diffusion coupled with advection and radiative transfer. The modelisation of phenomenon that affects such structures are described in the next paragraph.

\subsection{Finite element formulation of phenomena}

\subsubsection{Thermal diffusion}

Solving heat equation with finite element method is a well-known problem dealt in numerous publications. ${ }^{10,11}$ Heat equation and boundary conditions are written under the form (1). Finite element formulations of this equation can be found in. ${ }^{10}$

$$
\begin{cases}\rho c \frac{\partial T}{\partial t}=\nabla \cdot[k \nabla T]+q & \text { on the domain } \Omega \\ k \nabla T \cdot \vec{n}=\Phi_{s}+h_{c o n v}\left(T_{a}-T\right)+\varepsilon \sigma\left(T_{c}^{4}-T^{4}\right) & \text { at the surface } \\ k \nabla T \cdot \vec{n}=0 & \text { on the others boundaries }\end{cases}
$$

\subsubsection{Hydraulic convection}

We suppose the porous layer saturated in fluid. We note $\phi$ the porosity. Fluid flow answers to the Darcy law and its speed verifies : $u=\frac{-K \rho g}{\mu} \nabla\left(\frac{p}{\rho g}+z\right)$. Two temperature fields are used: $T$ for the solid, defined in the entire domain and $T_{f}$ for the fluid, only defined on the porous domain.

Heat equation written at a microscopic scale, and use of a representative volume element (RVE) allows to get a coupled equation system involving RVE's average values of both temperature fields. This coupled system is written $^{12,13}$ :

$$
\begin{aligned}
(1-\phi) \rho c \frac{\partial T}{\partial t} & =(1-\phi) \nabla \cdot[k \nabla T]+h_{f s} a_{f s}\left(T_{f}-T\right) \\
\phi \rho_{f} c_{f} \frac{\partial T_{f}}{\partial t}+\rho_{f} c_{f} \vec{u} \cdot \nabla T_{f} & =\phi \nabla \cdot\left[k_{f} \nabla T_{f}\right]+h_{f s} a_{f s}\left(T-T_{f}\right)
\end{aligned}
$$

With $h_{f s}$ an exchange coefficient verifying ${ }^{13} \frac{1}{h_{f s}}=\frac{d_{P}}{N u_{f s} k_{f}}+\frac{d_{P}}{\beta k}$ and $a_{f s}$ the specific surface given with $a_{f s}=\frac{6(1-\phi)}{d_{P}} . d_{P}$ is the size of granulates, $N u_{f s}$ the Nsselt number and $\beta$ a constant.

Boundary condition are of Dirichlet or Neumann type. We note $\Gamma_{f, D}$ and $\Gamma_{f, N}$ respectively the boundaries submitted to Dirichlet and Neumann conditions for the fluid temperature field and $\Gamma_{s, D}$ and $\Gamma_{s, N}$ respectively the boundaries submitted to Dirichlet and Neumann conditions for the solid-phase temperature on the porous domain.

The boundary conditions are defined as :

$$
\begin{cases}T=T_{D} \text { on } \Gamma_{s, D} & T_{f}=T_{f, D} \text { on } \Gamma_{f, D} \\ k \nabla T=h_{s}\left(T_{N}-T\right)+\Phi \text { on } \Gamma_{s, N} & k_{f} \nabla T_{f}=h_{f}\left(T_{f, N}-T_{f}\right)+\Phi_{f} \text { on } \Gamma_{f, N}\end{cases}
$$

To introduce the finite element formulation of equations (2) and (3), we note $\Omega_{f}$ the domain composed of fluid and porous layer in which the porous layer is submitted to convective exchange with the fluid, $H^{k}$ the Sobolev space of polynomial functions of degree $k$ on $\Omega_{f},\langle\cdot \mid \cdot\rangle_{\Omega_{f}}$ the scalar product for $H^{k}$ in $\Omega_{f}$ and $\langle\cdot \mid\rangle_{\Gamma, N i n}$ the scalar product for $H^{k}$ in $\partial \Gamma, N$ where - refers to the fluid phase $f$ or the solid phase $s$. The two scalar products are defined, for all $v, w \in H^{k}$ :

$$
\langle v \mid w\rangle_{\Omega_{f}}=\int_{\Omega_{f}} v w \mathrm{~d} \Omega,\langle v \mid w\rangle_{\Gamma_{\cdot, N}}=\int_{\Gamma_{\cdot, N}} v w \mathrm{~d} S
$$


Applying the Galerkin method on equations (2) and (3) allows to write, for all $\varphi \in H^{k}$ :

$$
\left\{\begin{array}{l}
\left.\left\langle(1-\phi) \rho c \frac{\partial T^{h}}{\partial t} \mid \varphi\right\rangle_{\Omega_{f}}+\left\langle h_{f s} a_{f s} T^{h} \mid \varphi\right\rangle_{\Omega_{f}}+\left\langle(1-\phi) k \nabla T^{h} \mid \nabla \varphi\right\rangle_{\Omega_{f}}\right\rangle_{\Omega_{f}}+\left\langle(1-\phi) h_{s} T^{h} \mid \varphi\right\rangle_{\Gamma_{N}} \\
=\left\langle h_{f s} a_{f s} T_{f}^{h} \mid \varphi\right\rangle_{\Omega_{f}}+\left\langle(1-\phi)\left(h_{s} T_{N}+\Phi\right) \mid \varphi\right\rangle_{\Gamma_{N}} \\
\left\langle\phi \rho_{f} c_{f} \frac{\partial T_{f}}{\partial t} \mid \varphi\right\rangle_{\Omega_{f}}+\left\langle\rho_{f} c_{f} \vec{u} \cdot \nabla T_{f}^{h} \mid \varphi\right\rangle_{\Omega_{f}}+\left\langle h_{f_{s}} a_{f s} T_{f}^{h} \mid \varphi\right\rangle_{\Omega_{f}}+\left\langle\phi k_{f} \nabla T_{f}^{h} \mid \nabla \varphi\right\rangle_{\Omega_{f}}+\left\langle\phi h_{f} T_{f}^{h} \mid \varphi\right\rangle_{\Gamma_{f, N}} \\
=\left\langle h_{f s} a_{f s} T^{h} \mid \varphi\right\rangle_{\Omega_{f}}+\left\langle\phi\left(h_{f} T_{f, N}+\Phi_{f}\right) \mid \varphi\right\rangle_{\Gamma_{f, N}}
\end{array}\right.
$$

Where $T^{h}$ and $T_{f}^{h}$ are the finite element approximations of $T$ and $T_{f}$ on $H^{k}$.

Because of the advection term $\vec{u} \cdot \nabla T_{f}^{h}$, the second equation of system (6) is hyperbolic. Solving this equation with the finite element method can entail spurious oscillations, particularly for the case of high Péclet number. To avoid this problem, we have chosen to use the Petrov-Galerkin formulation for this equation. The form function $\varphi$ is changed to $\varphi+\delta \vec{u} \cdot \nabla \varphi$ for the scalar product in $\Omega_{f}$ to introduce numerical diffusion. ${ }^{14}$ Some choices are available for $\delta$. Many authors ${ }^{15-17}$ suggest to use for $\delta$ a function of $\frac{h}{2\|\vec{u}\|}$ and the Péclet number. As an example, we can use:

$$
\delta=\frac{h}{2\|\vec{u}\|}\left(\operatorname{coth}(P e)-\frac{1}{P e}\right)
$$

Because we use in the following parts linear elements, the term $\left\langle\phi k \nabla T_{f}^{h} \mid \delta \vec{u} \cdot \nabla \varphi\right\rangle_{\Omega_{f}}$ can be neglected.

\subsubsection{Radiative transfer in semi-transparent layer}

We present in this part the modeling of thermal diffusion and radiative transfer coupling effects. This semitransparent pavement is quite transparent for solar radiation, and quite opaque for high wavelength radiation. Radiative transfer in this layer answer to radiative transfer equation. Knowing the optical properties of the structure, we can compute the intensity field $I_{\nu}$ in the semi-transparent domain.

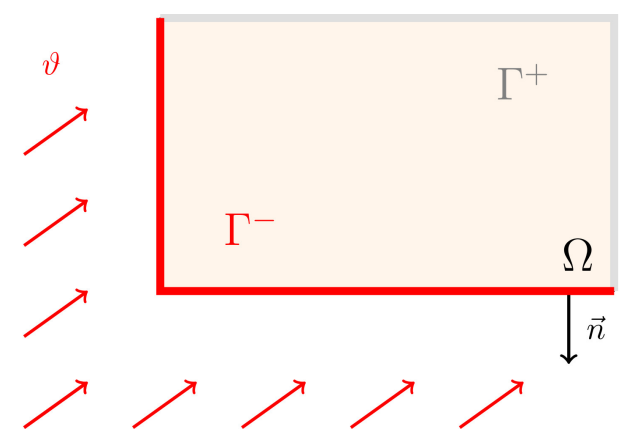

Figure 3: Input boundaries for a given direction

The radiative transfer equation is written, $\forall(s, \vec{\vartheta}) \in \Omega \times S_{2}$, for a given frequency $\nu$ :

$$
\vec{\vartheta} \cdot \nabla I_{\nu}(s, \vec{\vartheta})=-\left(\kappa_{\nu}+\sigma_{\nu}\right) I_{\nu}(s, \vec{\vartheta})+\kappa_{\nu} B_{\nu}(T(s))+\frac{\sigma_{\nu}}{4 \pi} \int_{4 \pi} p_{\nu}(\tilde{\vec{\vartheta}}, \vec{\vartheta}) I_{\nu}(s, \tilde{\vec{\vartheta}}) \mathrm{d} S
$$

Boundary conditions are written on the input boundary, described in figure 3 , with two components: outside coming radiation $g_{\text {out }}$ and reflective intern radiation $g_{\text {in }}$ : 


$$
I_{\nu}(s, \vec{\vartheta})=g_{\text {out }}(s, \vec{\vartheta})+g_{\text {in }}\left(I_{\nu}, s, \vec{\vartheta}\right)
$$

Some methods are usually used to solve the radiative transfer equation as finite volumes, Monte-Carlo or discrete ordinates. ${ }^{18,19}$ We propose here to focus on the finite element method as introduced by ${ }^{20,21}$ then coupling this solving with thermal diffusion and hydraulic convection solving. Using the finite element method for radiative transfer also allows us to keep the same spatial mesh to solve the other problems. Two discretizations are performed: for directions, within a unit sphere, and for space. Directional discretization is realized with discrete ordinate method. For a directional mesh having $N_{S}$ elements with nodes $\vec{\vartheta}_{i}$ and weights $w_{i}$, integral expressions can be rewritten, for any variable $u$ continuous on the unit sphere $\int_{4 \pi} u(\vec{\vartheta}) \mathrm{d} S=\sum_{i=1}^{N_{S}} w_{i} u\left(\vec{\vartheta}_{i}\right)$. This discretization is equivalent to a discontinuous Galerkin method with constant interpolation functions on the elements. In order to get a mesh having directions distributed the most uniformly on the unit sphere, we propose to use a discretization based on an regular icosahedron evoked in. ${ }^{21}$ Each direction is oriented to the centroid of the triangular faces. Weights are proportional to the area of faces, so they are all equals. By default, the number of directions is also equal to 204 . To further refine this mesh, each triangular face can be divided into four new triangular faces, projected on the unit sphere 5 . To get a more refined mesh, this process can be iterated.

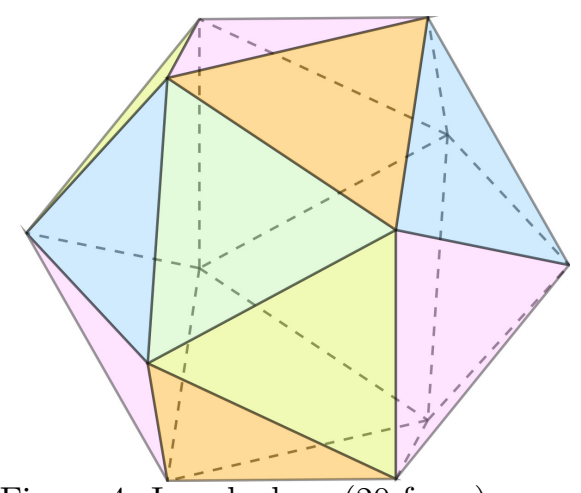

Figure 4: Icosahedron (20 faces)

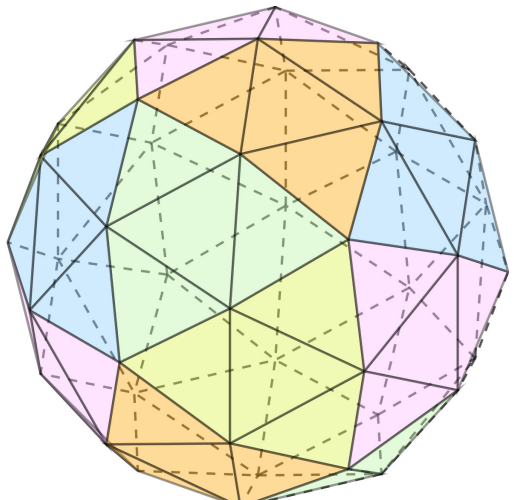

Figure 5: 80 faces polyhedron got from an icosahedron

By applying the discrete ordinates method, we obtain a system of $N_{S}$ equations from (8) and (9) :

$$
\left\{\begin{array}{l}
\vec{\vartheta}_{k} \cdot \nabla I_{\nu}\left(s, \vec{\vartheta}_{k}\right)=-\left(\kappa_{\nu}+\sigma_{\nu}\right)+\kappa_{\nu} B_{\nu}(T(s))+\frac{\sigma_{\nu}}{4 \pi} \sum_{l=1}^{N_{S}} w_{l} p_{\nu}\left(\vec{\vartheta}_{l}, \vec{\vartheta}_{k}\right) I_{\nu}\left(s, \vec{\vartheta}_{l}\right) \forall(s, k) \in \Omega \times\left[\left|1, N_{S}\right|\right] \\
I_{\nu}\left(s, \vec{\vartheta}_{k}\right)=g_{\text {out }}\left(s, \vec{\vartheta}_{k}\right)+g_{\text {in }}\left(I_{\nu}, s, \vec{\vartheta}_{k}\right)\left(s, \vec{\vartheta}_{k}\right) \in \Gamma^{-}
\end{array}\right.
$$

We introduce the following notations:

- $W_{\Omega}=\left\{u \in L^{2}(\Omega) / \forall \vec{\vartheta} \in S_{2}, \vec{\vartheta} \cdot \nabla u \in L^{2}\left(\Omega \times S_{2}\right)\right\}$

- $\partial \Omega_{k}^{-}=\left\{s \in \partial \Omega\right.$ such as $\left.\vec{n}(s) \cdot \vec{\vartheta}_{k}<0\right\}$

- $W_{0, k}=\left\{u \in L^{2}\left(\partial \Omega_{k}^{-}\right)\right\}$

We define scalar products on $\Omega$ and $\partial \Omega_{k}^{-}$denoted by $\langle\cdot \mid \cdot\rangle_{\Omega}$ and $\langle\cdot \mid \cdot\rangle_{k^{-}}$and defined as: 


$$
\begin{aligned}
\left\langle a_{1} \mid b_{1}\right\rangle_{\Omega} & =\int_{\Omega} a_{1}(s) b_{1}(s) \mathrm{d} \Omega, a_{1}, b_{1} \in W_{\Omega} \\
\left\langle a_{2} \mid b_{2}\right\rangle_{k^{-}} & =\int_{\partial \Omega_{k}^{-}} a_{2}(s) b_{2}(s) \mathrm{d} \sigma, \forall a_{2}, b_{2} \in W_{0, k}
\end{aligned}
$$

The system of radiative transfer equations can be rewritten, for each direction $\vartheta_{k}$ of the directional mesh, under the weak form ${ }^{20,22}$ :

$$
\forall \varphi \in W_{\Omega},\left\{\begin{array}{l}
\left\langle\mathcal{A}_{\nu} I_{\nu}\left(\cdot, \vec{\vartheta}_{k}\right) \mid \varphi\right\rangle_{\Omega}=\left\langle f_{k} \mid \varphi\right\rangle_{\Omega} \\
\left\langle\mathcal{B}_{\nu} I_{\nu}\left(\cdot, \vec{\vartheta}_{k}\right) \mid \varphi\right\rangle_{k^{-}}=0
\end{array}\right.
$$

Because radiative transfer equation is a hyperbolic partial differential equation, as for the advection equation (3), solving system (13) can lead to produce spurious variations. To get a physic numerical solution, we need to use a stabilization method, as the Petrov-Galerkin formulation introduced in section 2.2.2. Using PetrovGalerkin formulation allows to rewrite System (13) as:

$$
\forall \varphi \in W_{\Omega},\left\{\begin{array}{l}
\left\langle\mathcal{A}_{\nu} I_{\nu}\left(\cdot, \vec{\vartheta}_{k}\right) \mid \varphi+\delta \vec{\vartheta} \cdot \nabla \varphi\right\rangle_{\Omega}=\left\langle f_{k} \mid \varphi\right\rangle_{\Omega} \\
\left\langle\mathcal{B}_{\nu} I_{\nu}\left(\cdot, \vec{\vartheta}_{k}\right) \mid \varphi\right\rangle_{k^{-}}=0
\end{array}\right.
$$

With $\delta$ the streamline diffusion parameter, depending on the local mesh size and the coefficients $\sigma_{\nu}$ and $\kappa_{\nu}$. Both formulations (13) and (14) are equivalent.

For a spatial mesh with $N_{\Omega}$ nodes, we get a linear system with $N_{S} \times N_{\Omega}$ unknowns for each frequential interval which is written:

$$
\left(T_{h}+M_{h}\left(\kappa_{\nu}\right)+S_{h}\left(\sigma_{\nu}\right)+R_{h}\left(\rho_{\nu}\right)\right) I_{\nu}=M_{h}\left(\kappa_{\nu}\right) B_{\nu}+Q_{h} G_{\nu}
$$

Where $T_{h} I_{\nu}$ represents the transport effects, $M_{h}\left(\kappa_{\nu}\right) I_{\nu}$ the absorption losses, $S_{h}\left(\sigma_{\nu}\right) I_{\nu}$ the scattering effects, $R_{h}\left(\rho_{\nu}\right)$ the reflective effects, $M_{h}\left(\kappa_{\nu}\right) B_{\nu}$ the emission gain and $Q_{h} G_{\nu}$ the outside contribution.

The source term can be divided into two parts: a volumetric source $S_{v o l}$ coming from radiation absorption inside the semi-transparent material and a flux $S_{\text {flux }}$ reaching the interfaces between opaque and semi-transparent medium. These two components can be written ${ }^{19}$ :

$$
\begin{aligned}
S_{v o l} & =-\nabla \cdot\left[\int_{\nu=0}^{+\infty} \int_{4 \pi} I_{\nu}(s, \vec{\vartheta}) \vec{\vartheta} \mathrm{d} S \mathrm{~d} \nu\right] \\
S_{\text {flux }} & =\int_{\nu=0}^{+\infty}\left[\left(1-\rho_{\nu}\right) \int_{\vec{\vartheta} \cdot \vec{n}>0} I_{\nu}(s, \vec{\vartheta}) \vec{\vartheta} \cdot \vec{n} \mathrm{~d} S-\varepsilon_{\nu} \pi B_{\nu}(T)\right] \mathrm{d} \nu
\end{aligned}
$$

\subsection{Numerical modeling}

We solve the problem for the mesh shown in figure 6 . The semi-transparent layer is colored in grey and the porous layer in blue. We model the surface of the pavement as smooth.

The thermal properties of subdomains are written in table 1 . The fluid velocity in the porous layer is determined from an outflow measurement. We obtain $\|\vec{u}\|=3.0 \mathrm{~mm} \cdot \mathrm{s}^{-1}$.

The environmental conditions, air temperature $T_{a}$, sky temperature $T_{s}$, input fluid temperature $T_{f, \text { in }}$ and convective exchange coefficient $h_{\text {conv }}$, are defined in table 2 . 


\begin{tabular}{lcccc}
\hline & layer 1 & layer 2 & layer 3 & fluid (water) \\
\hline$k\left[\mathrm{~W} . \mathrm{m}^{-1} \cdot \mathrm{K}^{-1}\right]$ & 0.85 & 1.03 & 1.40 & 0.60 \\
$\rho c\left[\mathrm{~J} . \mathrm{K}^{-1} \cdot \mathrm{m}^{-3}\right]$ & $2.27 \times 10^{6}$ & $1.84 \times 10^{6}$ & $2.25 \times 10^{6}$ & $4.18 \times 10^{6}$ \\
\hline \multicolumn{4}{r}{ Table 1: Thermal properties of the materials }
\end{tabular}

\begin{tabular}{cccc}
\hline$T_{a}\left[{ }^{\circ} \mathrm{C}\right]$ & $T_{s}\left[{ }^{\circ} \mathrm{C}\right]$ & $T_{f, i n}\left[{ }^{\circ} \mathrm{C}\right]$ & $h_{\text {conv }}\left[\mathrm{W} \cdot \mathrm{m}^{-2} \cdot \mathrm{K}^{-1}\right]$ \\
\hline 30 & 24 & 24 & 5.7
\end{tabular}

Table 2: Environmental conditions

The heat flux incident to the surface of the semi-transparent layer is computed from data of figure 14 for steady state. For the optical properties, we suppose that the layer is opaque for wavelengths higher than $2.7 \mu m$. Below this value, we have $\sigma=10 \mathrm{~m}^{-1}$ for scattering and $\kappa=20 \mathrm{~m}^{-1}$ for absorption.

The temperature field at the surface of the semi-transparent layer is shown in figure 7 .

To solve this coupled problem, the finite element method is applied to thermal diffusion (system (1)), thermal exchanges in the porous layers (equations (2) and (3)) and radiative transfer in the semi-transparent layer (equation (8)) with linear elements as it was described in previous sections.

The numerical scheme is the following: at each time step, we first compute the radiative intensity field in the semi-transparent layer, then we determine the thermal source terms from equations (16) and (17). These terms are introduced in thermal diffusion and advection equations as a heat source. For the time dependence, we use a Crank-Nicholson scheme.

\section{THERMAL MONITORING BY INFRARED THERMOGRAPHY}

\subsection{Measurements considerations and thermal infrared calibration}

A laboratory experiment has been conducted by monitoring our mock-up through an infrared camera, thermocouples sensors, a meteorological station and a pyranometer (Figure 13). The objective of such monitoring is to show the possibilities of a non destructive, low cost and robust solution. The signal received by the infrared camera gives an information on the radiated thermal energy, emitted by the surface of an object. The flux received by the thermal camera will depend on the environmental conditions during the experiment and the thermo-optics properties of the measurement scene. ${ }^{24,25}$ Therefore, the radiation flux attenuated by the atmosphere depends on the measurement point in the scene, as shown in Figure 8. For the particular case of our experiment, the environment radiation is neglected compared to our source radiation: a local spot lamp that simulates the sun effect.

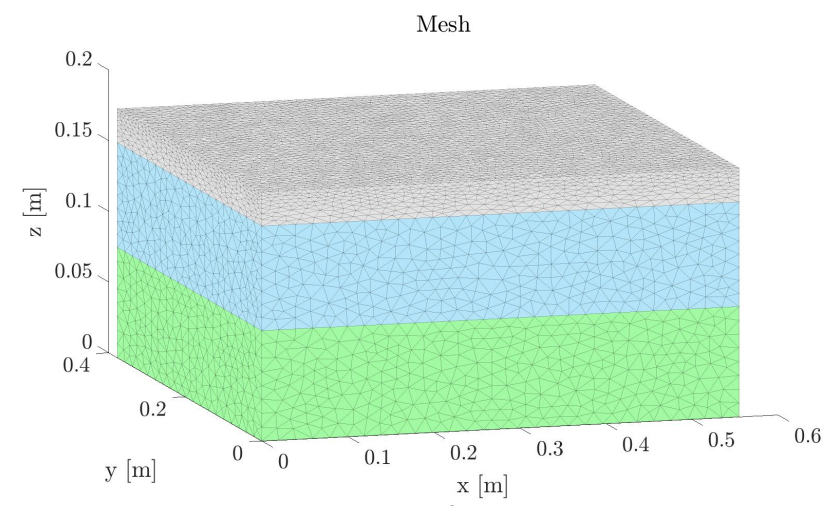

Figure 6: Mesh of the structure

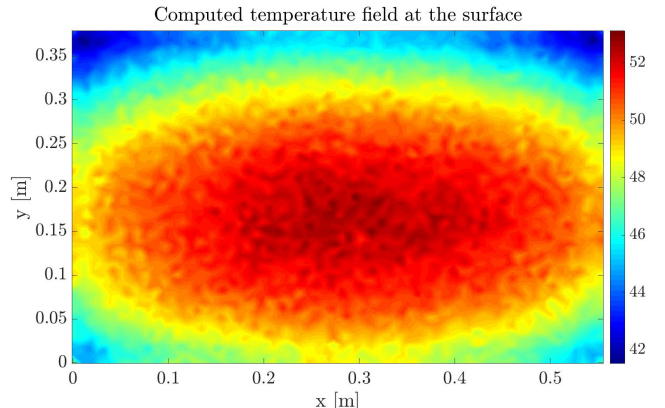

Figure 7: Surface temperature field for steady state 


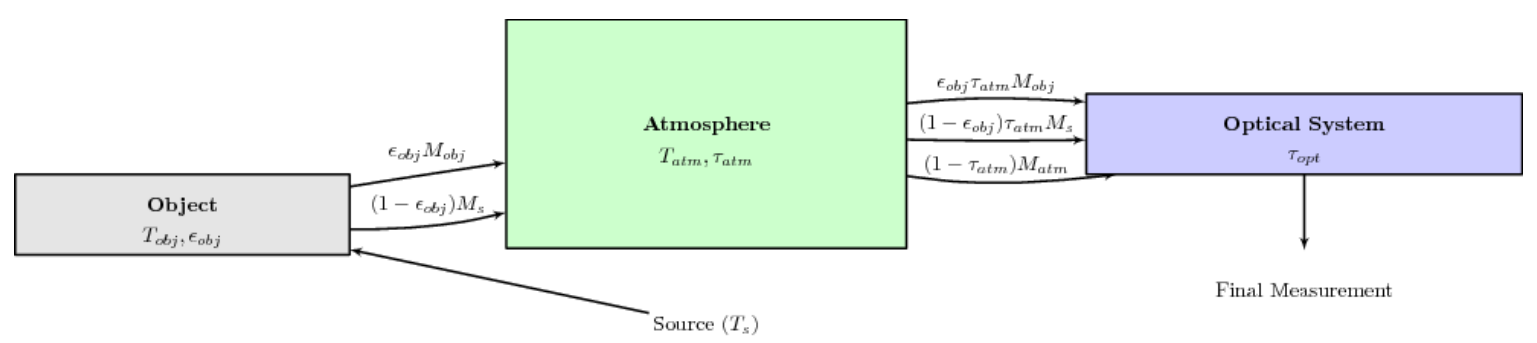

Figure 8: Interaction between the emitted radiation and its environment.

A first approximation is to derive the total radiant exitance $L_{\text {total }}$ through the simplified radiative transfer equation 18. This way, we can retrieve the value of the flux received by the camera for every points of the infrared image.

$$
L_{\text {total }}^{i, j}=\epsilon_{o b j} \tau_{a t m} \tau_{o p t} L_{o b j}^{i, j}+\left(1-\epsilon_{o b j}\right) \tau_{a t m} \tau_{o p t} L_{s}^{i, j}+\left(1-\tau_{a t m}\right) \tau_{o p t} L_{a t m}
$$

Please note that in the equation 18 , every physical quantity that does not have a $i, j$ exposant is considered as constant for a given scene. Otherwise, the $i, j$ exposant shows that we are dealing with matrices in the formula. Since the experiment occurs indoor in specific conditions, the contribution of the atmosphere in the the previous equation has been neglected. Moreover, we have considered an ideal case where $\tau_{\text {opt }}=1$. Such approximations lead us to the equation 19.

$$
L_{\text {total }}^{i, j}=\epsilon_{o b j} L_{o b j}^{i, j}+\left(1-\epsilon_{o b j}\right) L_{s}^{i, j}
$$

From this equation, we can solve $L_{o b j}^{i, j}$ which is the radiant exitance equivalent to a black body with the same temperature as the object. Finally, we get the object temperature from the rbf function 20:

$$
T_{o b j}^{i, j}=\frac{B}{\log \left(\frac{R}{L_{o b j}^{i, j}}+F\right)}
$$

A black body calibration source Mikron M310 $\left(\epsilon_{e f f}=1, \forall \lambda \in[8 \mu \mathrm{m}, 14 \mu \mathrm{m}]\right)$ has been used for thermally calibrating the infrared camera. Experimental calibration at $10 \mathrm{~K}$ sampling have been made, considering the temperature range involved during the experiment $\left(T_{o b j}^{i, j} \in[293.15 K, 343.15 K]\right)$. The $R, B$ and $F$ coefficients are finally found by minimizing $\left(T_{o b j}-T_{\text {calibration }}\right)^{2}$ with the Levenberg-Marquardt algorithm (9). Results of such calibration is shown on Figure 10.

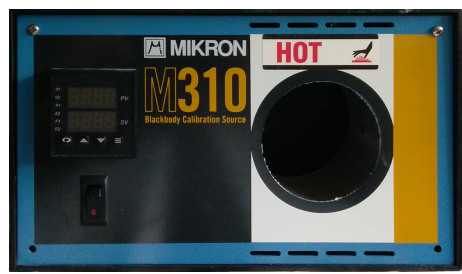

(a) Mikron M310 blackbody calibration source

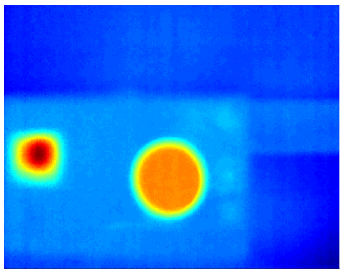

(b) Infrared image of the calibration source

Figure 9: Thermal calibration of infrared cameras. 


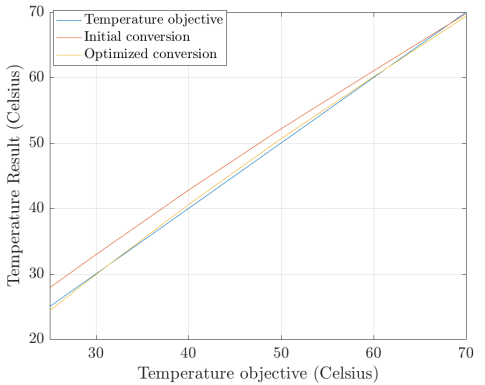

(a) Calibration optimization result

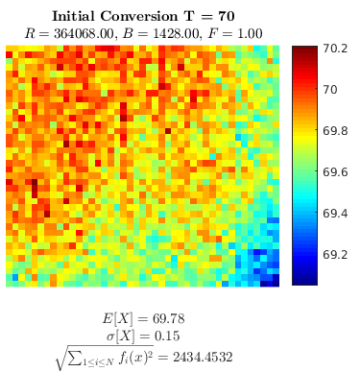

(b) Calibration differences example at $70^{\circ} \mathrm{C}$

Figure 10: Thermal calibration results

\subsection{Spatial calibration of infrared images}

The resolution of the simplified radiative transfer equation in every points of the infrared image requires the knowledge of the spatial parameters that depends on the scene-sensor configuration. In fact, due to the field of view of the camera, the spatial sampling is not constant in the observed scene. In order to identify those spatial parameters, a calibration has been set up by applying the "Gold Standard" algorithm by R. Hartley and A. Zisserman. ${ }^{26}$ Moreover, in order to compare the numerical model with the experimental results, it was necessary to rectify the camera projection, select the region of interest and finally resize the image to be consistent with the numerical mesh. The input points are measured on the mock-up and their corresponding points on the image selected through a supervised and user-friendly method. We can define the calibration matrix $M$ such as for every couple $(x, X) \in \mathbb{R}^{2} \times \mathbb{R}^{3}$ of corresponding points between the observed image and the real world, we have:

$$
x=M X
$$

We can derive the matrix $M$ in homogeneous coordinates such as:

$$
M=\left(\begin{array}{ccc}
f & s_{k} & c_{x} \\
0 & a f & c_{y} \\
0 & 0 & 1
\end{array}\right)\left(\begin{array}{cccc}
1 & 0 & 0 & 0 \\
0 & 1 & 0 & 0 \\
0 & 0 & 1 & 0
\end{array}\right)\left(\begin{array}{cc}
R_{3 \times 3} & 0_{3 \times 1} \\
0_{1 \times 3} & 1
\end{array}\right)\left(\begin{array}{cc}
I_{3} & T_{3 \times 1} \\
0_{1 \times 3} & 1
\end{array}\right)
$$

In order to solve this system, we need the prior knowledge of 6 points coordinates. Those 6 points will be normalized such as:

- The barycenter will be at the origin of the frame.

- In average, the points will be around the unit ball of there space in euclidian norm.

From this, we define two normalization matrices $U$ and $V$ :

$$
\hat{X}_{i}=U X_{i}, \quad \hat{x}_{i}=V x_{i}
$$

Finally, the Levenberg-Marquardt algorithm is used to minize the geometric distance error defined by equation $24^{27,28}$

$$
\min \sum_{1 \leq i \leq N} d\left(\hat{x}_{i}, M \hat{X}_{i}\right)
$$


In order to minimize this error, the jacobian matrix ${ }^{29}$ is necessary and evaluated through its analytical form. From equation 21 written in homogeneous coordinates:

$$
\left(\begin{array}{c}
s u \\
s v \\
s
\end{array}\right)=M\left(\begin{array}{c}
X \\
Y \\
Z \\
1
\end{array}\right)
$$

Therefore, the jacobian matrix is expressed as:

$$
J_{M}=\frac{\partial(\tilde{u}-u, \tilde{v}-v, 1-s)}{\partial\left(f, a, c_{x}, c_{y}, s_{k}, t_{x}, t_{y}, t_{z}, w_{1}, w_{2}, w_{3}\right)}
$$

where $T=\left(t_{x}, t_{y}, t_{z}\right)$ and $\left(w_{x}, w_{y}, w_{z}\right)$ is obtained by the Rodrigues' rotation formula. ${ }^{30}$ The flowchart of the algorithm is presented in figure 11

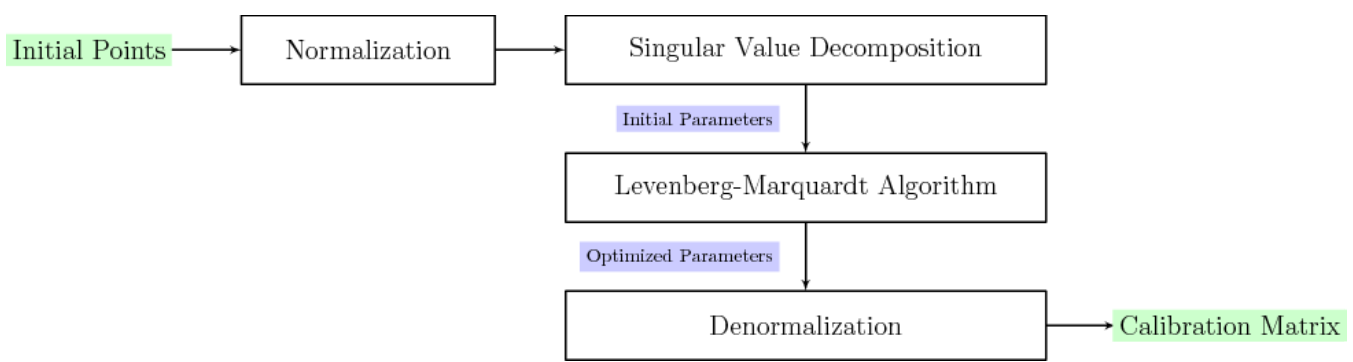

Figure 11: Spatial calibration algorithm

A distance map and an angle map are then deducted from this calibration. Once calibrated, the image is adjusted to recover a non projective view and focus on a region of interest.

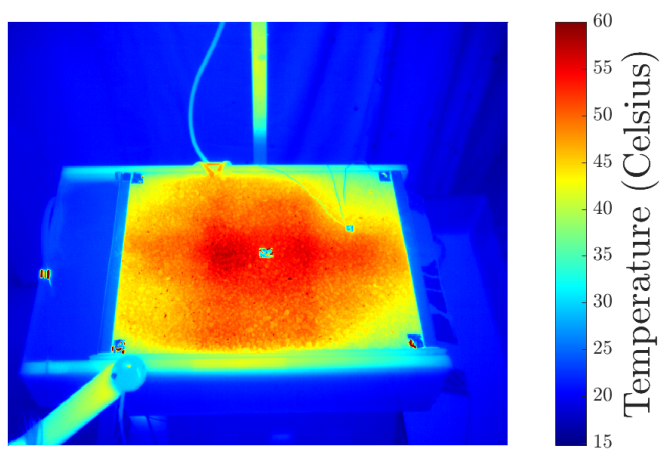

(a) Original image converted to temperature

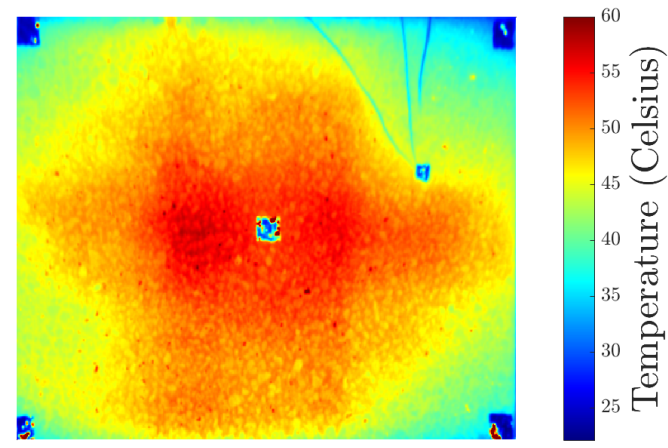

(b) Image converted to temperature after spatial calibration, region of interest selection and resizing

Figure 12: Comparison of the infrared measurement before and after spatial calibration process and ROI selection.

\section{EXPERIMENTS AND RESULT ANALYSIS}

\subsection{Experimental set-up}

The measurements have been made on a mock-up composed of 3 layers as explained in figure 2. A closed circuit driven by a pump makes water flowing from tank 1 to tank 2 in the second layer (figure 13). A 1500 watts 
halogen lamp at $3000 \mathrm{~K}$ is simulating the sun radiation. Four thermocouples have been put on the mock-up to give back information on the temperature at different points: the roads surface, the water temperature in the two tanks and at the interface between the pavement and the porous layers. The infrared images are obtained by a FLIR A65 camera (spectral range of 7.5 - $13 \mu \mathrm{m}$ ), calibrated as explained in 3.1. Finally, a meteorological station and a pyranometer measure the relative humidity, ambient temperature and the solar radiation flux density.

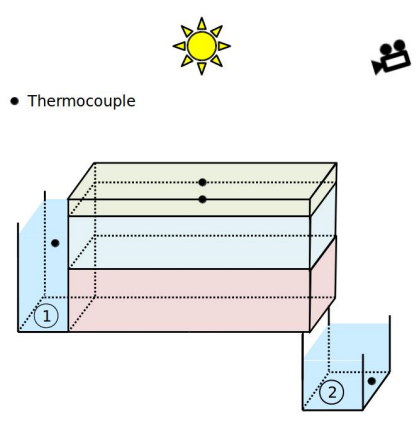

(a) Schematic view of the mock-up

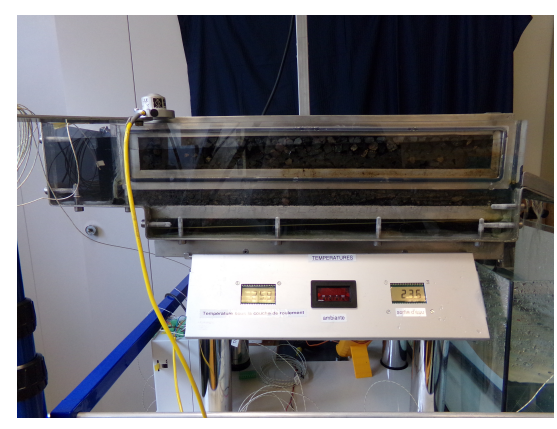

(b) Solar hybrid road laboratory mock-up

Figure 13: Laboratory mock-up

The radiant exitance source term on the surface model is obtained by correlating the value returned by the pyranometer sensor to a calibration map, computed prior to the experiment. The calibration map is obtained by performing a bicubic interpolation on nine measurements points, in green in figure 14 .

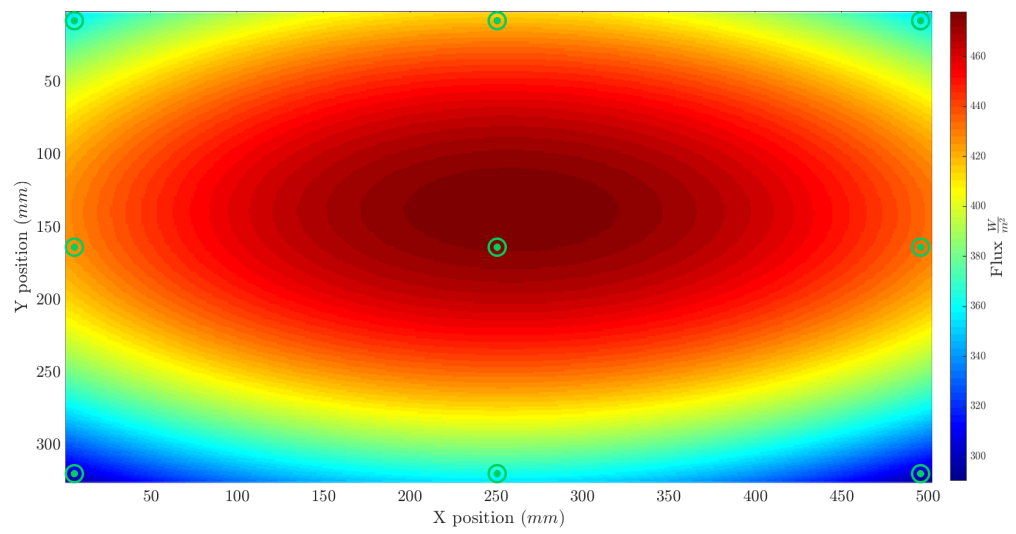

Figure 14: Pyranometer calibration map

\subsection{Comparison with numerical simulations}

The previous selection of the region of interest enabled us to easily compare the theoretical model with the measurements. The infrared images have been averaged through the time by manually identifying the steady state of the system (see figure 15), generating one image to compare with the numerical simulations.

Since the theoretical model does not take into account the material texture, a gaussian filter has been applied to the infrared measurement to cut down the high frequencies to make the comparison as fair as possible. As we can see in figure $12 \mathrm{~b}$, control points made with reflective adhesive tapes are outliers for the comparison and have to be removed from the measurements. This pre-processing step is achieved by smoothing the image first with a gaussian kernel on a guided filter ${ }^{31}$ to preserve edges. A median filter is applied to the resulting image. This median filter enable us to uniformize the image's areas while preserving the edges. A canny edge detection $^{32}$ is then applied to the images gradient. Finally Otsu's histogram thresholding ${ }^{33}$ and a morphological 


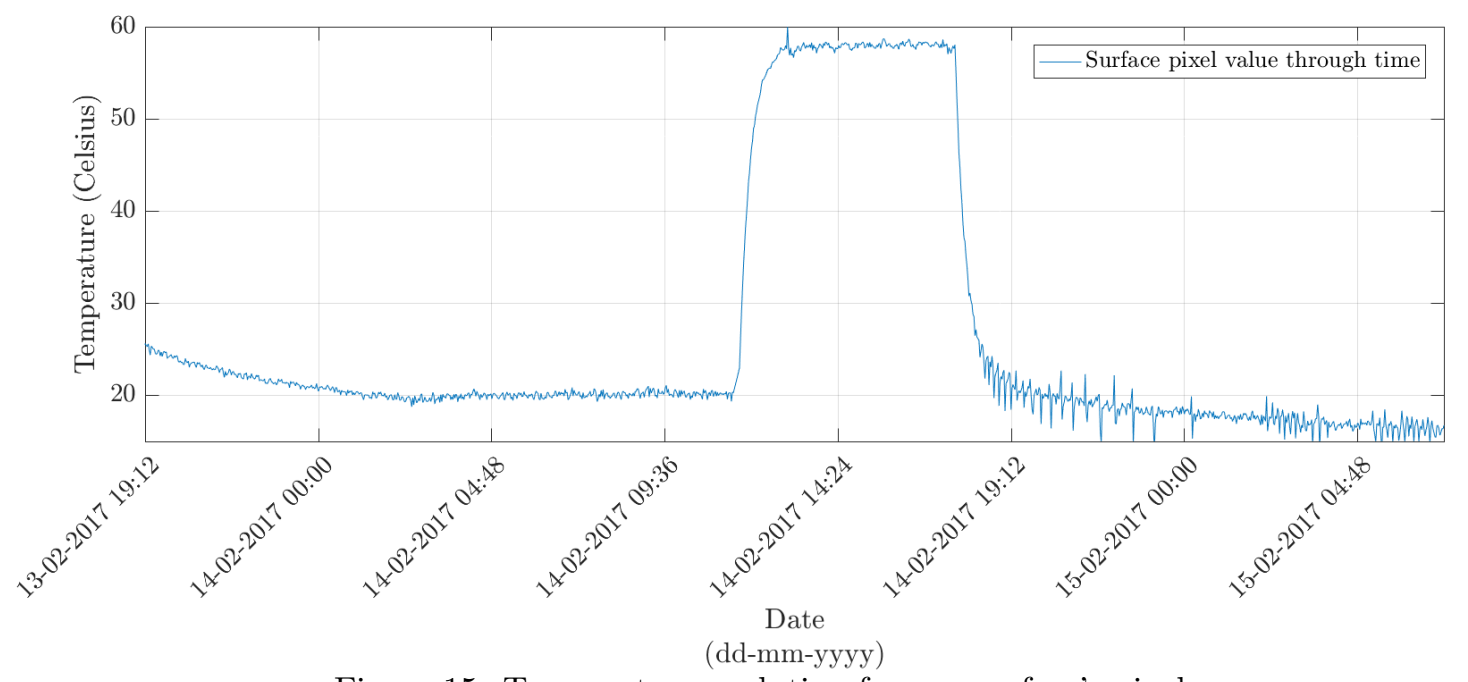

Figure 15: Temperature evolution for one surface's pixel.

close operation give us the final result 16. We can note that if we want a more accurate result on the detected areas, we could add at the end a few iterations of an active contour algorithm. This final step was not necessary in this study.

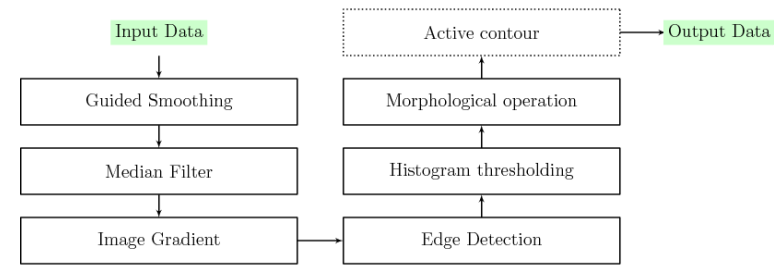

(a) Pre-processing step flowchart

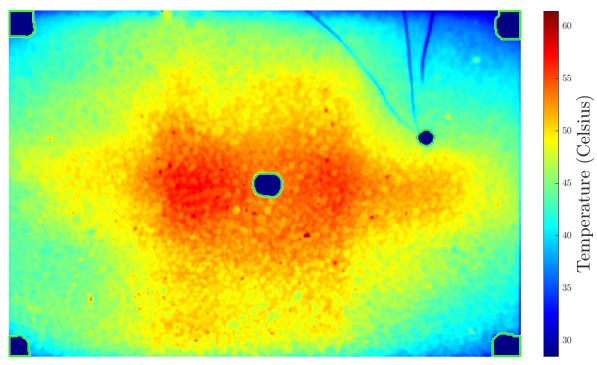

(b) Outliers detected and removed from the infrared image

Figure 16: Pre-processing

The comparison of the numerical values and the measurements is given in figure 17. An absolute difference of $2.98 \mathrm{~K}$ is observed on the overall surface, with a $2.34 \mathrm{~K}$ standard deviation. However, when we focus more at the center, this average becomes $1.41 \mathrm{~K}$ with $0.95 \mathrm{~K}$ standard deviation. Those differences can be explained by multiple factors. First of all, the emissivity of the materials surface is not well known and only estimated. Such estimation has an impact on the numerical model but also on the temperature computation from the infrared measurement. In fact, the natural texture of the coated layer will produce a larger emissivity and a high variation of local emissivities values that should be considered during the computation of the temperature. Moreover, the geometrical properties of the scene and thus the interpolations performed to settle the model create bias on the temperature conversion process. The mock-up is also composed of photovoltaic panels at the interface of the two layers that is not considered in the numerical model.

\section{CONCLUSION AND PERSPECTIVES}

In this study, we have analyzed two methods to get the temperature field at the surface of a semi-transparent pavement having a porous layer in which a fluid flows. 


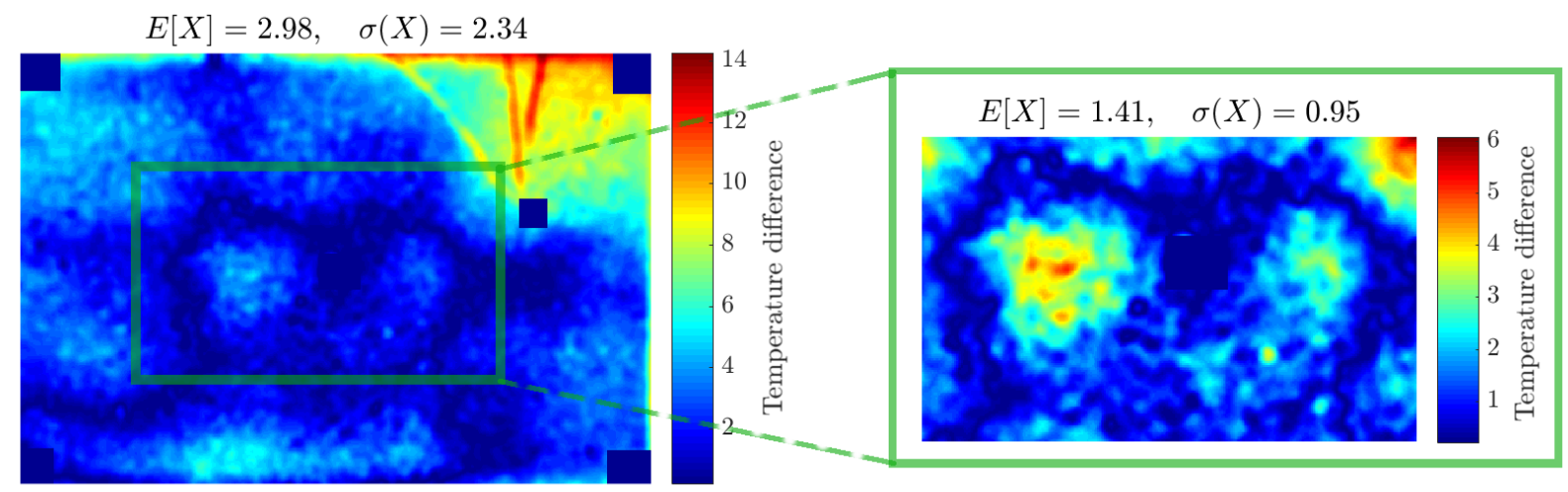

Figure 17: Absolute difference between the numerical simulation and the measurements. Surface temperature with numerical simulation is obtained from a linear interpolation. Average and standard deviation are given on the overall surface and at the center.

First, we have presented the equations that govern heat diffusion, hydraulic convection and radiative transfer. Then we have applied the finite element method to discretize the problem. For hydraulic convection and radiative transfer, which are advection equations, a Petrov-Galerkin method is used to stabilize the problem solving.

Then, we have conducted a laboratory experiment using an instrumented mock-up with an infrared camera. The assumptions behind the model used to convert the numerical values of the camera to temperature has been discussed. The calibration techniques both thermal and spatial for the camera has been presented. Once calibrated, the image has been recovered to settle the numerical model with a pre-processing algorithm.

A comparison of the results obtained both with numerical simulation and infrared thermography was then led. In steady case, with a lamp acting as a direct solar flux, we get similar temperature fields at the center of the surface structure. Differences of temperature are a little more important close to the edges.

Even if non negligible differences exist, those preliminary results are encouraging. In fact, we have provided a numerical model and a low-cost, non-destructive monitoring method through infrared thermography. The temperature conversion model of the infrared measurements can be improved by incorporating data collected by our sensors and use the radiative transfer equation model 18. Moreover, the numerical simulation could be refined by knowing the surface layer material properties and in particular its texture. Finally, the lamp creates defects on the surface that can be seen in figure 17 with the two spots at the center. This is due to the reflectors of the lamp and has an impact on the comparison.

A next step will be the study of this kind of structure for a non-steady state case.

\section{ACKNOWLEDGMENTS}

The authors wish to thank Stéphane Bouron, Andrea Themeli, Emmanuel Chailleux, Jean-Michel Piau, Alain Grosseau, Jean-Philippe Gourdon, Fabrice Blaineau and Jean-Luc Manceau for their technical support during the building of the hybrid solar road mock-up, that was initially exposed at the French pavillon for the COP21 during 2015 Paris Climate Conference.

\section{REFERENCES}

[1] Duarte, F. and Ferreira, A., "Energy harvesting on road pavements: state of the art," Proceedings of the institution of civil engineers 169, 79-90 (2016).

[2] Beeby, S. P., Tudor, M. J., and White, N. M., "Energy harvesting vibration sources for microsystems applications," Meas. Sci. Technol. 17, R175-R195 (2006).

[3] Zhao, H., Yu, J., and Ling, J., "Finite element analysis of cymbal piezoelectric transducers for harvesting energy from asphalt pavement," Joournal of the Ceramic Society of Japan 118(10), 909-915 (2010). 
[4] Arroyo, E. and Badel, A., "Electromagnetic vibration energy harvesting device optimization by synchronous energy extraction," Sensors and actuators A 171, 266-273 (2011).

[5] Mallick, R. B., Chen, B.-L., and Bhowmick, S., "Harvesting energy from anphalt pavements and reducing the heat island effect," International Journal of Sustainable Engineering 2(3), 214-228 (2009).

[6] Van Bijsterveld, W. T., Houben, L. J. M., Scarpas, A., and Molenaar, A. A. A., "Using pavement as solar collector: Effect on pavement temperature and structural response," Transportation Research Record Journal of the Transportation Research Board 1778, 140-148 (2001).

[7] Bobes-Jesus, V., Pascual-Muoz, P., Castro-Fresno, D., and Rodriguez-Hernandez, J., "Asphalt solar collectors: a literature review," Applied Energy 102, 962-970 (2013).

[8] Asfour, S., Bernardin, F., Toussaint, E., and Piau, J.-M., "Hydrothermal modeling of porous pavement for its surface de-freezing," Applied Thermal Engineering 107, 493-500 (2016).

[9] "Modeling snow melting on heated pavement surfaces. part i: Model development," Applied Thermal Engineering 27, 115-1124.

[10] Gartling, D. K. and Reddy, J. N., [The finite element in heat transfer and fluid dynamics], CRC Press, Boca Raton, 3rd ed. (2010).

[11] Taine, J., Enguehard, F., and Iacona, E., [Transferts thermiques, introduction aux transferts d'nergie], Dunod, Paris, 5th ed. (2014).

[12] Bejan, A., Dincer, I., Lorente, S., Miguel, A. F., and Reis, A. H., [Porous and complex flow structures in modern technologies], Springer Science, New York (2004).

[13] Nield, D. A. and Bejan, A., [Convection in porous media], Springer Science, New York, fourth ed. (2013).

[14] Johnson, C., [Numerical solutions of partial differential equations by the finite element method], Cambridge University Press, Cambridge (1987).

[15] Donea, J. and Huerta, A., [Finite Element Methods for Flow Problems], John Wiley \& Sons, Ltd., Chichester (2003).

[16] Codina, R., [Comparison of some finite element methods for solving the diffusion-convection-reaction equation], vol. 156 (1998).

[17] Brooks, A. N. and Hughes, T. J. R., "Streamline upwind/petrov-galerkin formulations for convection dominated flows with particular emphasis on the incompressible navvier-stokes equations," Computer Methods in applied mechanics and engineering 32, 199-259 (1982).

[18] Howell, J. R. and Siegel, R., [Thermal radiation heat transfer], CRC Press, Boca Raton, fifth ed. (2010).

[19] Modest, M. F., [Radiative heat transfer].

[20] Kanschat, G., "Solution of radiative transfer problems with finite elements," in [Numerical methods in multidimensional radiative transfer], Kanschat, G., Meinkhn, E., Rannacher, R., and Wehrse, R., eds., Springer-Verlag, Berlin (2009).

[21] Richling, S., Meinkhn, E., Kryzhevoi, N., and Kanschat, G., "Radiative transfer with finite element. i. basic method and tests," $A \& A$ 380, 776-788 (2001).

[22] Meinkohn, E., Kanschat, G., Rannacher, R., and Wehrse, R., "Numerical methods for multidimensional radiative transfer," in [Reactive flow, diffusion and transport], Jger, W., Rennacher, R., and Warnatz, J., eds., Springer-Verlag, Berlin (2007).

[23] Incropera, F. P. and DeWitt, D. P., "Fundamentals of heat and mass transfer," (1990).

[24] Dumoulin, J. and Boucher, V., "Infrared thermography system for transport infrastructures survey with inline local atmospheric parameter measurements and offline model for radiation attenuation evaluations," Journal of Applied Remote Sensing 8(1), 084978-084978 (2014).

[25] Howell, J., Siegel, R., and Pinar, M., [Thermal Radiation Heat Transfer.], CRC Press, 5th ed. (2010).

[26] Hartley, R. and Zisserman, A., [Multiple View Geometry in Computer Vision], Cambridge University Press, Cambridge, UK ; New York, 2 edition ed. (Apr. 2004).

[27] Chong, E. K. P. and Zak, S. H., [An Introduction to Optimization], Wiley-Blackwell, Hoboken, New Jersey, 4th edition ed. (Feb. 2013).

[28] Pérez Muñoz, J., Ortiz Alarcón, C., Osorio, A., Mejía, C., and Medina, R., "Environmental applications of camera images calibrated by means of the Levenberg-Marquardt method," Computers 8 Geosciences 51, 74-82 (Feb. 2013). 
[29] Marquardt, D. W., "An algorithm for least-squares estimation of nonlinear parameters," Journal of the society for Industrial and Applied Mathematics 11(2), 431-441 (1963).

[30] Fillmore, J. P., "A note on rotation matrices," IEEE Computer Graphics and Applications 4(2), 30-33 (1984).

[31] He, K., Sun, J., and Tang, X., "Guided Image Filtering," IEEE Transactions on Pattern Analysis and Machine Intelligence 35, 1397-1409 (June 2013).

[32] Canny, J., "A Computational Approach to Edge Detection," IEEE Transactions on Pattern Analysis and Machine Intelligence PAMI-8, 679-698 (Nov. 1986).

[33] Otsu, N., "A Threshold Selection Method from Gray-Level Histograms," IEEE Transactions on Systems, Man, and Cybernetics 9, 62-66 (Jan. 1979). 